\title{
COVID-19 lockdown presented a chance to evaluate emergency referrals to paediatric neurosurgical unit: a prospective cohort study
}

\author{
Ahmed Aly ${ }^{1}$ (D) $\cdot$ Benedetta Pettorini ${ }^{1}$ \\ Received: 20 October 2020 / Accepted: 23 November 2020 / Published online: 6 January 2021 \\ (C) Springer-Verlag GmbH Germany, part of Springer Nature 2021
}

\section{Dear Editor:}

At the peak of the COVID-19 lockdown period in the UK [1] and especially in April 2020, the neurosurgical team at Alder Hey Children's Hospital noticed a significant decrease in acute referrals. In our neurosurgical unit, we act as the regional tertiary referral centre for the northwest, and even a supraregional referral centre for some craniofacial and vascular pathologies. We decided to audit the referrals which we keep the records of on a prospective referral documentation system, and we decided to compare the period from March to May between 2019 and 2020.

\section{Methods}

Data were collected from a prospective referral documentation system that we use in $\mathrm{AHCH}$. As the normal acute referral process will usually start by a call and bleep to the on-call neurosurgical registrar/fellow, who in turn would take the referral and document all the information during the call on the referral system. The compliance for referral documentation on this database is reviewed weekly on the department MDT meeting. Also the referral protocol in the unit has not changed due to the COVID-19 pandemic; thus, it was not a confounding factor.

The documentation includes the demographics, history, clinical examination, radiological investigations, and the advice of the neurosurgical registrar to the caller. Also,

Ahmed Aly

ahmedhma88@gmail.com

1 Paediatric Neurosurgery Department, Alder Hey Children's Hospital, Liverpool, UK the outcome is categorised, and the approval of the consultant on-call is documented as well. By extracting these data to an Excel sheet, we carried out the analysis to compare the referrals' total volumes as well as looking in details into the nature of pathologies and outcomes of these referrals.

\section{Results}

The total number of referrals in March to May 2019 was 189 compared to 127 referrals in the same period in 2020 . Comparing the individual months (Fig. 1) shows a drop of referrals volume in March 2020 with 27\% less referrals than March 2019. This is followed by a bigger $58 \%$ drop of number of referrals in April 2020 compared to same month in 2019. In May 2020, the referral number picks up again to approach the volume in May 2019 with only 7\% less referrals.

When we start analysing the pathologies/diagnoses of these referrals (Table 1), it becomes obvious that the most drop in referral volume is mainly due to significant less referrals with 'no neurosurgical problem identified' label as the outcomes (24 less referral over the whole comparison period), as well as to lesser extent less trauma referrals (11 referrals less). The differences in absolute number of referrals over the 3-month period comparison between 2019 and 2020 are represented in (Fig. 2). In regard to CSF-related referrals, the total numbers over the 3-month comparison period were comparable between 2019 and 2020 ( 32 and 30 respectively). That is despite the fact that the CSF referrals showed a marked drop in April 2020 in comparison with April 2019, but the difference is compensated in March and May (Fig. 3). 


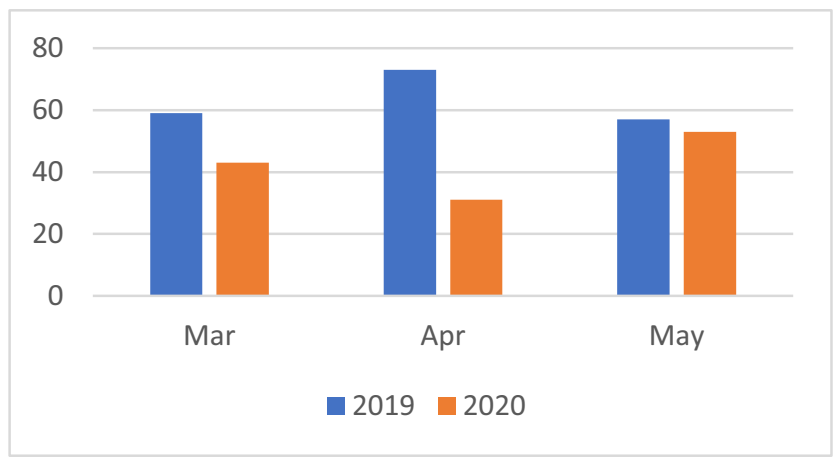

Fig. 1 Total numbers of referrals in March-May in 2019 and 2020

Table 1 Numbers of referrals according to diagnosis/pathology in March-May in 2019 versus 2020

Fig. 2 The differences in absolute numbers of referrals according to diagnosis/pathology

\begin{tabular}{llllll}
\hline 2019 & & & & & \\
& March & April & May & Total & \\
No Nsx Dx & 14 & 23 & 8 & 45 & $24 \%$ \\
Trauma & 14 & 17 & 21 & 52 & $28 \%$ \\
CSF & 12 & 14 & 6 & 32 & $17 \%$ \\
Infection & 6 & 2 & 2 & 10 & $5 \%$ \\
Congenital anomalies & 6 & 5 & 7 & 18 & $10 \%$ \\
Vascular & 4 & 4 & 4 & 12 & $6 \%$ \\
Seizures & 1 & & & 1 & $1 \%$ \\
Tumour & 1 & 4 & 6 & 11 & $6 \%$ \\
CF & 1 & 1 & 1 & 3 & $2 \%$ \\
CCJ & & 1 & 2 & 3 & $2 \%$ \\
Degenerative spine/MSK & & 2 & & 2 & $1 \%$ \\
2020 & & & & & \\
& March & April & May & Total & \\
No Nsx Dx & 8 & 9 & 4 & 21 & $17 \%$ \\
Trauma & 10 & 5 & 26 & 41 & $32 \%$ \\
CSF & 14 & 7 & 9 & 30 & $24 \%$ \\
Infection & 1 & & 2 & 3 & $2 \%$ \\
Congenital anomalies & 1 & 6 & 2 & 9 & $7 \%$ \\
Vascular & 3 & 2 & & 5 & $4 \%$ \\
Seizures & & & & 0 & $0 \%$ \\
Tumour & 1 & 2 & 2 & 5 & $4 \%$ \\
CF & 1 & & 5 & 6 & $5 \%$ \\
CCJ & 2 & & 2 & 4 & $3 \%$ \\
Degenerative spine/ MSK/ peripheral nerves & 2 & & 1 & 3 & $2 \%$ \\
\hline
\end{tabular}

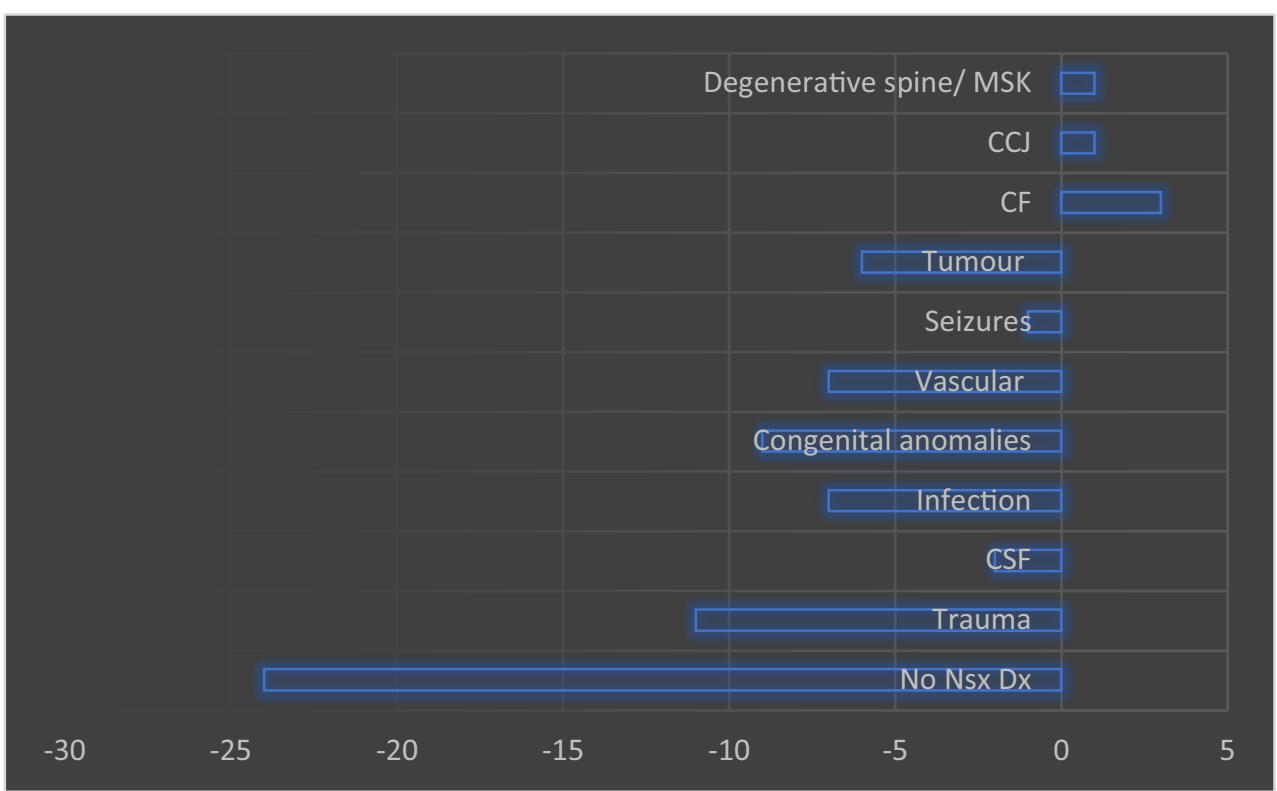


Fig. 3 Numbers of CSF referrals in March-May of 2019 versus 2020

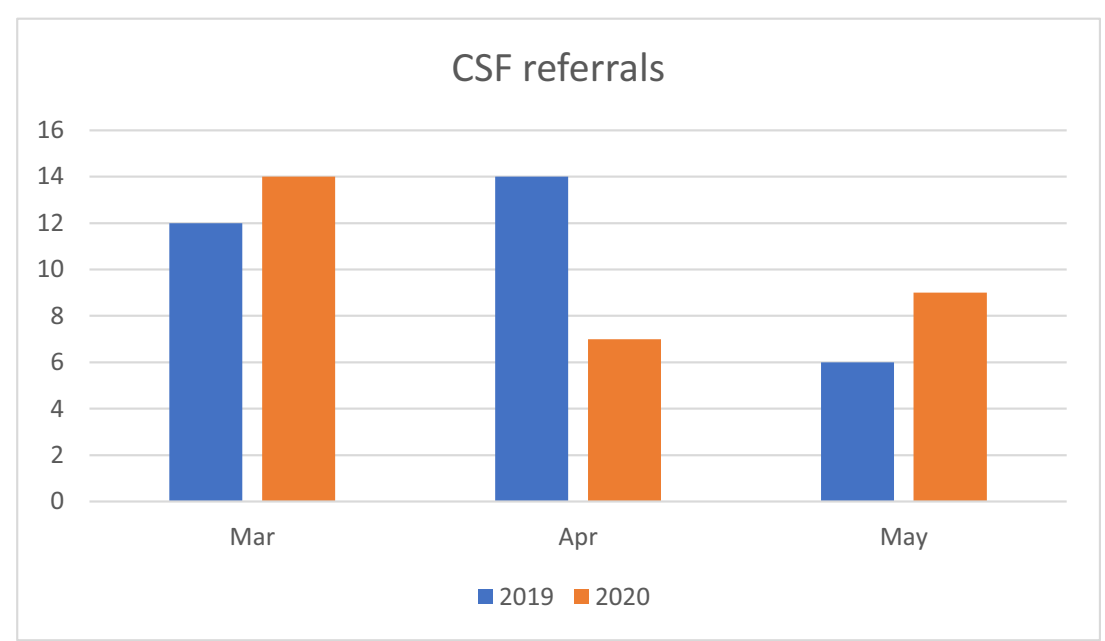

After diving to analyse the outcome/advice of the referrals (Table 2) (Fig. 4), one can see that in 2020 a smaller percentage of patients are taken in for admission in AHCH than the same period in 2019. The difference in admission percentage has gone to more outpatient appointments and redirection to other departments when no intervention is needed from $\mathrm{AHCH}$ neurosurgery team directly.

\section{Discussion}

There was a marked reduction of acute referrals made to on-call neurosurgery team at $\mathrm{AHCH}$ during the COVID19 lockdown period. This quick audit of referral data helped to prove it as well as to help us to understand the possible reasons of this drop. Despite the subjective observations from the on-call registrars that there were less CSF-related referrals, for example, it became clear that this was not necessarily true. The observation about the drop of CSF referral volume happened in April 2020, but when including the whole period of the peak of lockdown (March-May), we concluded that the numbers

Table 2 Numbers of referrals in March-May of 2019 vs 2020 according to outcomes

\begin{tabular}{|c|c|c|c|c|c|c|c|c|}
\hline & \multicolumn{4}{|l|}{2019} & \multicolumn{4}{|l|}{2020} \\
\hline & March & April & May & Total & March & April & May & Tota \\
\hline Taken in & 11 & 24 & 19 & 54 & 9 & 4 & 11 & 24 \\
\hline Redirected & 26 & 35 & 14 & 75 & 20 & 17 & 17 & 54 \\
\hline OPA & 22 & 14 & 24 & 60 & 14 & 10 & 25 & 49 \\
\hline
\end{tabular}

were comparable to 2019. The actual major drops in referrals during the lockdown was attributed to markedly less referrals with 'no neurosurgical problem' outcome followed by a drop in trauma related referrals.

The drop in these referrals with the eventual outcome of 'no neurosurgical problem' can be easily explained by overall reduction of $\mathrm{A} \& \mathrm{E}$ attendances all over the country [1-4]. Examples of referrals with 'no neurosurgical problem' outcome are incidental findings on scans done for other reasons (and do not need surgical input), known neurosurgical patients who presented to A\&E with other issues (e.g. chicken pox) and babies with scan findings unfamiliar to treating physicians. It has been always a problem with these kind of referrals to the on-call registrars, which should prompt future audits and studies to probe the reasons behind the shear numbers of these referrals. The drop in trauma referrals was also expected due to the lockdown with less outdoor activity of the population, even though a recent letter by Dyson et al. [5] to child's nervous system showed an increase of trauma cases at Great Ormond Street Hospital's paediatric neurosurgical department.

Also, the percentage of these referrals during the COVID19 lockdown period who have been accepted as inpatients were reduced from $29 \%$ in 2019 to $19 \%$ in 2020 over the 3month comparison period. The difference of admission percentage was majorly shifted to the pool of patients who got outpatients appointments, as the percentage of patients who were redirected to other centres, specialities, or their local hospitals was comparable between 2019 and 2020 (40\% versus $43 \%$, respectively).

In conclusion, it is interesting to see how much the drop in acute referrals with no ultimate neurosurgical issue causes a significant drop in the burden to the highly specialised paediatric neurosurgery unit at $\mathrm{AHCH}$. This should be further 
Fig. 4 Percentage of referrals outcomes in March-May of 2019 vs 2020

studied and audited in order to optimise the referral systems, thus, to allow the team to deliver more benefit to the patients who need their attention and service more.

Data availability Anonymous data used in this analysis are available from main author on reasonable request.

\section{Compliance with ethical standards}

Conflict of interest The authors declare that they have no conflict of interest.

Code availability Not applicable.

\section{References}

1. Cabinet Office Staying at home and away from others (social distancing)
2. Isba R, Edge R, Auerbach M, Cicero M.X., Jenner R., Setzer E., Broughton E., Keegan T. (2020) COVID-19: transatlantic declines in pediatric emergency admissions. Pediatric Emergency Care Publish Ahead of Print: https://doi.org/10.1097/PEC. 0000000000002260, Publish Ahead of Print

3. Callan R, Assaf N, Bevan K (2020) Impact of the COVID-19 pandemic on acute general surgical admissions in a district general hospital in the United Kingdom: a retrospective cohort study. Surgery Research and Practice 2020:1-7. https://doi.org/10.1155/2020/ 2975089

4. NHS England A\&E attendances and emergency admissions March 2020 statistical commentary

5. Dyson EW, Craven CL, Tisdall MM, James GA (2020) The impact of social distancing on pediatric neurosurgical emergency referrals during the COVID-19 pandemic: a prospective observational cohort study. Childs Nerv Syst 36:1821-1823. https://doi.org/10.1007/ s00381-020-04783-4

Publisher's note Springer Nature remains neutral with regard to jurisdictional claims in published maps and institutional affiliations. 